\title{
Guidance and Control Algorithms for the Mars Entry, Descent and Landing Systems Analysis
}

\author{
Jody L. Davis ${ }^{*}$ and Alicia Dwyer Cianciolo ${ }^{\dagger}$ \\ NASA Langley Research Center, Hampton, VA, 23681 \\ Richard W. Powell ${ }^{\star}$ and Jeremy D. Shidner ${ }^{\S}$ \\ Analytical Mechanics Associates, Inc., Hampton, VA, 23666 \\ and \\ Eduardo García-Llama** \\ GBTech, Inc., Houston, TX, 77058
}

\begin{abstract}
The purpose of the Mars Entry, Descent and Landing Systems Analysis (EDL-SA) study was to identify feasible technologies that will enable human exploration of Mars, specifically to deliver large payloads to the Martian surface. This paper focuses on the methods used to guide and control two of the contending technologies, a mid- lift-to-drag (L/D) rigid aeroshell and a hypersonic inflatable aerodynamic decelerator (HIAD), through the entry portion of the trajectory. The Program to Optimize Simulated Trajectories II (POST2) is used to simulate and analyze the trajectories of the contending technologies and guidance and control algorithms. Three guidance algorithms are discussed in this paper: EDL theoretical guidance, Numerical Predictor-Corrector (NPC) guidance and Analytical Predictor-Corrector (APC) guidance. EDL-SA also considered two forms of control: bank angle control, similar to that used by Apollo and the Space Shuttle, and a center-of-gravity (CG) offset control. This paper presents the performance comparison of these guidance algorithms and summarizes the results as they impact the technology recommendations for future study.
\end{abstract}

\section{Nomenclature}

$\begin{array}{ll}\text { ALHAT } & \text { Autonomous Landing and Hazard Avoidance Technology } \\ \mathrm{CG} & =\text { center of gravity } \\ \mathrm{EDL} & =\text { Entry Descent and Landing } \\ \mathrm{EDL}-\mathrm{SA} & =\text { Entry Descent and Landing - Systems Analysis } \\ \mathrm{HIAD} & =\text { Hypersonic Inflatable Aerodynamic Decelerator } \\ \mathrm{L} / \mathrm{D} & =\text { lift-to-drag ratio } \\ \mathrm{POST} 2 & =\text { Program to Optimize Simulated Trajectories II } \\ \mathrm{X}_{\mathrm{CG}} & =\text { axial center-of-gravity location } \\ \mathrm{Y}_{\mathrm{CG}} & =\text { lateral center-of-gravity location } \\ \mathrm{Z}_{\mathrm{CG}} & =\text { vertical center-of-gravity location } \\ \mathrm{X}_{\mathrm{CG}} / \mathrm{D} & =\text { axial center-of-gravity location over diameter } \\ \Delta \mathrm{V} & =\text { instantaneous change in velocity (delta velocity) }\end{array}$

\footnotetext{
* Aerospace Engineer, Atmospheric Flight \& Entry Systems Branch/D205, MS 489, AIAA Member.

${ }^{\dagger}$ Aerospace Engineer, Atmospheric Flight \& Entry Systems Branch/D205, MS 489, Senior AIAA Member.

* Senior Aerospace Engineer, Analytical Mechanics Associates, Inc, MS 489, AIAA Associate Fellow.

$\S$ Aerospace Engineer, Analytical Mechanics Associates, Inc, MS 489, AIAA Member.

${ }^{* *}$ Aerospace Engineer, GBTech, Aeroscience and Flight Mechanics Division/EG5.
} 


\section{Introduction}

$\mathrm{T}$

HE Mars Entry, Descent and Landing Systems Analysis (EDL-SA) main objective in FY09 was to identify feasible candidate technologies that will support human exploration of Mars, delivering payloads between 10 and $40 \mathrm{mt}$, to the surface. Two candidate aeroshell technologies for the Mars atmospheric entry portion of the trajectory are a $10 \mathrm{~m} \times 30 \mathrm{~m}$ mid- lift-to-drag (L/D) rigid aeroshell and a $23 \mathrm{~m}$ diameter hypersonic inflatable aerodynamic decelerator (HIAD). These technologies were chosen to compare the more traditional rigid body aeroshell with an inflatable technology that is currently undergoing testing. The purpose of the first year was to compare these two aeroshell technologies and determine the additional technologies required that would provide the aerocapture phase for cargo delivery vehicles and the EDL required by both cargo and crewed vehicles at Mars. The following summarizes the guidelines and requirements put in place for the Mars EDL-SA first year study: 1) the hyperbolic approach velocity was set at $7.36 \mathrm{~km} / \mathrm{s}, 2)$ the target orbit was a 1 Sol (33793km x $250 \mathrm{~km}$ ) Mars orbit, 3) EDL initiates from the $1 \mathrm{Sol}$ orbit, 4) the landing site is at zero m altitude, 5) touchdown provides $10 \mathrm{~m}$ accuracy and 6) the deceleration profiles remain within those limits set for a deconditioned crew. It was assumed for all the architectures that a reaction control system (RCS) would be the primary vehicle control for the bank angle control strategy, and CG movement in the vertical and lateral directions would be use for the center of gravity control cases. To emulate the characteristics of a RCS within a three-degree-of-freedom simulation, a "pseudo-controller" was used where the desired accelerations, rates, and control limits were modeled.

The main focus of this paper is to assess the performance of the different methods used to guide and control the two candidate technologies during atmospheric entry. The simulation used to analyze the trajectories of the candidate technologies and guidance and control algorithms is the Program to Optimize Simulated Trajectories II $(\text { POST2 })^{1}$. Three guidance algorithms were developed for the EDL-SA task: the EDL theoretical guidance, a Numerical Predictor-Corrector (NPC) guidance and an Analytical Predictor-Corrector (APC) guidance. The vehicles that represent these candidate technologies are large and the optimal control method is unknown. EDL-SA considered two forms of control, the first included bank angle control, similar to what is planned for the Mars Science Laboratory (MSL) mission, and the second considered a center-of-gravity (CG) offset control. This paper presents the performance comparison of the control methods evaluated, along with the three guidance algorithms, and summarizes the results as they impact the technology recommendations for future study.

\section{EDL-SA Simulation Overview}

The Mars EDL-SA POST2 simulation is a three-degree-of-freedom (3DOF) trajectory simulation used to assess the performance and feasibility of the candidate technologies as well as the performance of each of the different entry guidance and control strategies of these candidate technologies. POST2 is a generalized point mass, discrete parameter targeting and optimization trajectory simulation program used for mission and system development support, engineering trade studies, development of reference trajectories, and mission planning and operation support at NASA Langley Research Center. POST2 has the ability to simulate 3DOF, 6DOF, and multi-degree-offreedom trajectories for multiple vehicles, simultaneously, in various flight regimes. POST2 also has the capability to incorporate various gravity, atmosphere, terrain, vehicle, guidance, control, sensor and navigation system models.

Specific models used to tailor production POST2 for the EDL-SA purposes include use of the Mars Global Reference Atmosphere Model 2005 (MarsGRAM) ${ }^{2}$. The Mars planet model and terrain model that are being used for the Mars Science Laboratory were used for the EDL-SA simulation. Other EDL-SA simulation specific models include aerodynamic and aerothermodynamic models from NASA Ames Research Center for the two candidate aeroshell technologies: HIAD and mid-L/D rigid aeroshell, mass properties derived from response surface equations for HIAD and mid-L/D rigid aeroshell, a propulsion model, and the various guidance algorithms and control schemes discussed in this paper. In addition, EDL-SA assumes that the trajectories have perfect navigation knowledge and that technologies required to perform precise landings will be leveraged from the ALHAT project.

The EDL-SA POST2 EDL simulation consists of three phases. The first EDL phase, deorbit, begins with the POST2 simulation performing a deorbit $\Delta \mathrm{V}$ maneuver from the apoapsis of a 1 Sol elliptical orbit. The second phase, atmospheric entry, begins at a geographic radius of $3522.2 \mathrm{~km}$ and the Mars atmospheric model is activated. The guidance algorithms are initiated shortly after. The third phase, range control, is achieved by using either bank angle or CG control and guides the vehicle to the point that the propulsion system is activated. These phases summarize the entry portion of the trajectory included in the EDL-SA POST2 simulation. 
The EDL-SA POST2 simulation was used for both aerocapture and EDL analysis. Details of nominal reference trajectories and sensitivity studies for all architectures are presented elsewhere ${ }^{3}$. This paper focuses only on the simulation of the atmospheric entry portion of the trajectory.

\section{Entry Guidance Algorithms}

Several guidance algorithms were developed and used to design the atmospheric entry portions of the trajectory. The guidance algorithms considered assessed the feasibility of each of the candidate aeroshell technologies with acceptable margin, to maneuver during entry such that the vehicle could reach a specified target. Algorithms considered included a Numerical Predictor-Corrector (NPC) guidance, a theoretical guidance and an Apollo-derived Analytical Predictor-Corrector (APC) guidance. For entry analysis and mass optimization, the theoretical guidance was used as the baseline because of its ease of implementation and relatively quick computational time. However, to validate the EDL results, entry performance was compared to select simulations using non-theoretical flight guidance algorithms.

\section{A. Theoretical Guidance}

The "Theoretical EDL Guidance" was developed to be highly adaptable to support any of the EDL concepts. It was used primarily to develop the design trajectories such that the trajectories, mass model, and thermal models were all consistent. It also had the ability to be detuned such the results would be comparable to those expected from flight guidance algorithms, such that the design trajectories did not have to redone to support the actual guidance algorithms. It is POST2-based and has full knowledge of all environmental parameters such as aerodynamics, atmospheric density and wind profiles. The flight phases are representative of the numerical predictor-corrector and the Apollo-derived algorithms that were used in this study.

\section{B. Numerical Predictor-Corrector}

The Numerical Predictor-Corrector (NPC) algorithm ${ }^{4}$ was originally developed as candidate guidance for both the aerocapture and entry of the Mars Surveyor Program 2001 mission and the aerocapture of CNES Mars 2005 Sample Return Orbiter. The NPC algorithm operates using an outer loop called every simulation time step and an inner loop, called at some specified interval (e.g. every 10 seconds). For the EDL-SA implementation, the NPC receives perfect navigated state conditions (position and velocity) as well as current orientation and sensed bodyaxis accelerations. The algorithm integrates 3DOF translational equations of motion using a fourth-order RungeKutta integration scheme.

The NPC algorithm includes simplified models of the planet, gravity field, vehicle aerodynamics, body attitude, mass properties and atmosphere. The predictor corrector inner loop is activated on a deceleration trigger that is tailored for each candidate technology investigated. The algorithm produces a command vector that includes either commanded bank angle or commanded CG locations. For the bank angle control case, the bank angle magnitude is used to control total range, and the direction is used to control azimuth to the target. Thus, as the Space Shuttle does, it performs a series of bank reversals to maintain the azimuth error within the desire limits. A half-interval search method is used to determine the appropriate bank angle and reversal times. Sensed acceleration data is used to update the internally stored atmosphere density profiles and aerodynamics. This is done by calculating the ratios of sensed accelerations and comparing them to the values predicted by the guidance algorithm. By knowing the aerodynamic accelerations ratios, scalar multipliers to the guidance predicted aerodynamics and density may be calculated. These scalar multipliers and altitude bias quantities are averaged over 10 second intervals and the averages are used within the inner loop.

The guidance logic for the powered descent was developed to be a linear profile of velocity versus altitude, where the slope was specified. Once the vehicle intercepted this slope, the terminal descent phase was initiated.

\section{Analytical Predictor-Corrector}

The Analytic Predictor-Corrector (APC) used in the EDL-SA simulation is a combination of a modified Apollo entry guidance 5 and the Apollo powered descent guidance ${ }^{6}$. The modified Apollo entry guidance was a candidate guidance algorithm for both the aerocapture and entry of the Mars Surveyor Program 2001 mission, and the aerocapture of CNES Mars 2005 Sample Return Orbiter. It is the guidance for the Mars Science Laboratory mission, and will be the guidance for Orion return from the ISS. The modified Apollo powered descent guidance is currently under evaluation for Altair's lunar propulsive descent guidance.

The Apollo atmospheric entry guidance controls to a terminal range and velocity target using pre-derived influence coefficients with respect to errors about a nominal L/D reference trajectory which is defined by range-to- 
go, drag acceleration, and altitude rate as a function of relative velocity. The bank angle is reversed each time the out-of-plane central angle exceeds the reversal criterion, which is set as a quadratic function of the relative velocity. To minimize disturbance of downrange control near the target, bank reversals are inhibited below a given relative velocity magnitude. If the bank command is not in the same direction as the target at this point, then a final reversal is commanded prior to setting the inhibit flag. After that, a heading control phase is initiated. The reference trajectories basically consist of two segments. The first one, covering velocities approximately higher than $2 \mathrm{~km} / \mathrm{s}$, usually requires large bank angles to meet the constraints on heat load, heat rate, and deceleration load. The second segment, covering velocities approximately lower than $2 \mathrm{~km} / \mathrm{s}$, is designed with an increased vertical lift to further reduce the terminal relative velocity at a given terminal altitude. Vertical lift margin is provided in this segment in order to allocate dispersions. The end of the reference trajectory calls for a full lift-up configuration below the velocity at which the heading control phase is set to start.

The guidance logic for the powered descent phase is a quadratic guidance that uses a pre-stored gravity turn steering thrust reference profile that reaches the landing site at the touchdown conditions. It commands thrust and attitude to reduce position and velocity errors at touchdown.

The reference profile is a gravity turn trajectory that consists of three segments: a constant thrust level, followed by a ramp down, and a smaller constant thrust level. Two target points are determined for the whole trajectory, one is set at the end of the ramp down segment or few seconds before the end of the trajectory, and the other is set at the end of the trajectory, which ends at the predetermined final conditions.

\section{Entry Control Strategies}

Two different control strategies were used and analyzed in the EDL-SA study: bank angle control and CG offset control. The bank angle control strategy was used as the baseline along with the theoretical EDL guidance algorithm in the EDL-SA POST2 simulation for both the mid-L/D rigid aeroshell and HIAD entry vehicles. The other control method, CG offset control, was developed and analyzed as an alternative control strategy for the HIAD since bank angle control has not been established for inflatable entry vehicles. Initial studies show that the thruster sizes and fuel required for bank angle control were large, and the CG control was developed as an alternative.

\section{A. Bank Angle Control}

Bank angle control was the first method of control considered for the EDL-SA study, used for both the midL/D rigid aeroshell and HIAD. Bank angle accelerations of $5 \mathrm{deg} / \mathrm{s}^{2}$ and a max rate of $20 \mathrm{deg} / \mathrm{s}$ are used in the simulation and are based on many historical studies, and were found to provide adequate control for accurate landings. This strategy is comparable to that use by Apollo, MSL, and the Space Shuttle. Thus there is significant experience with bank angle control.

\section{B. CG Offset Control}

Center-of-gravity (CG) offset control was the alternate method considered for the HIAD technology because of the large thruster size and fuel requirements. Specifically, this control strategy consists of moving the HIAD CG location to produce direct angle-of-attack and sideslip control to provide the normal and side force necessary to meet the desired conditions at the start of terminal descent. Each CG channel is controlled separately and independently, with a fixed axial CG location $\left(\mathrm{x}_{\mathrm{CG}}\right)$, such that one component of the $\mathrm{CG}$ location controls angle-of-attack (vertical location or $\mathrm{z}_{\mathrm{CG}}$ ) and another component of the CG location controls sideslip angle (lateral location or $\mathrm{y}_{\mathrm{CG}}$ ). Figure 1 shows a plot of axial and vertical CG locations as a function of $\mathrm{L} / \mathrm{D}$ at the trim angle-of-attack. For axial locations further aft, the amount of movement is minimized to achieve the same L/D, because of the decrease in static stability. For this study, $0.2 \mathrm{~L} / \mathrm{D}$ performance was used as the CG control strategy baseline with an axial CG location $\left(\mathrm{x}_{\mathrm{CG}} / \mathrm{D}\right)$ of 0.35 . The $\mathrm{CG}$ movement for $\mathrm{CG}$

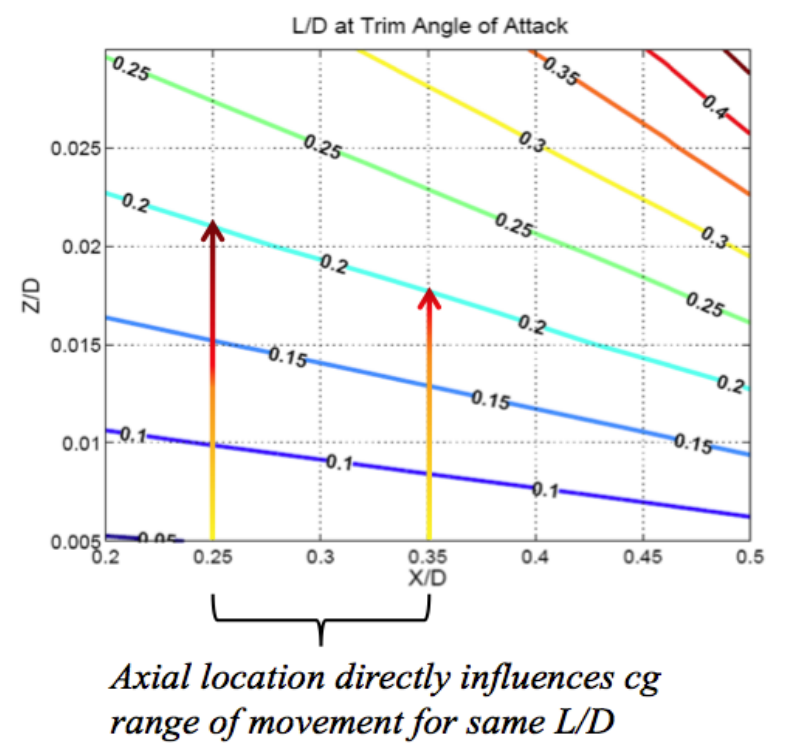

Figure 1. Axial CG location with $L / D$ at trim angle-ofattack. 
control is limited by performance with maximum movement rate of $0.0127 \mathrm{~m} / \mathrm{s}(0.5 \mathrm{in} / \mathrm{s})$ and an instantaneous maximum CG acceleration is assumed. CG control eliminates the open-loop bank reversal segments used in the bank angle control strategy since allowable heading error can be directly controlled by sideslip.

\section{Simulation Results}

Several POST2 nominal trajectories and Monte Carlos were run for each of the EDL-SA candidate aeroshell technology vehicle types, producing results to analyze the performance of each of these guidance and control methods discussed previously. Design parameters such as maximum deceleration, aerothermal heating rate and heating load and staging conditions (start of terminal descent engine initiation) helped determine and quantify system performance. Nominal trajectories and Monte Carlo results are presented and compared for the two control strategies and three different guidance algorithms.

\section{A. Guidance Comparison}

Monte Carlos with 2000-dispersed cases, varying atmosphere, aerodynamics, initial state and vehicle characteristics, were run using the EDL-SA POST2 simulation for the three different guidance algorithms. The comparison of the Monte Carlo simulations using APC, NPC and theoretical guidance algorithms for the mid-L/D aeroshell architecture is shown in Fig. 2. Both of the non-theoretical guidance algorithms (APC and NPC) can provide the required targeting accuracy of $10 \mathrm{~m}$ error at touchdown with perfect navigation and have minimal impact on the design parameters (maximum g-loading, maximum aerothermal heat rate and load, staging conditions, etc). The theoretical guidance performs well with respect to staging, or terminal descent engine initiation, in that

there are small

At Staging dispersions in altitude and Mach number. The nontheoretical guidance algorithms perform very well compared to the theoretical guidance, with NPC having a slightly larger dispersion in Mach number compared to APC and a smaller dispersion in altitude at staging due to the difference in staging event triggers. The propellant usage variations at touchdown for all the guidance algorithms are very similar. The results of the HIAD architecture Monte Carlos, shown in Fig. 3 , are similar in comparison to the mid-L/D aeroshell
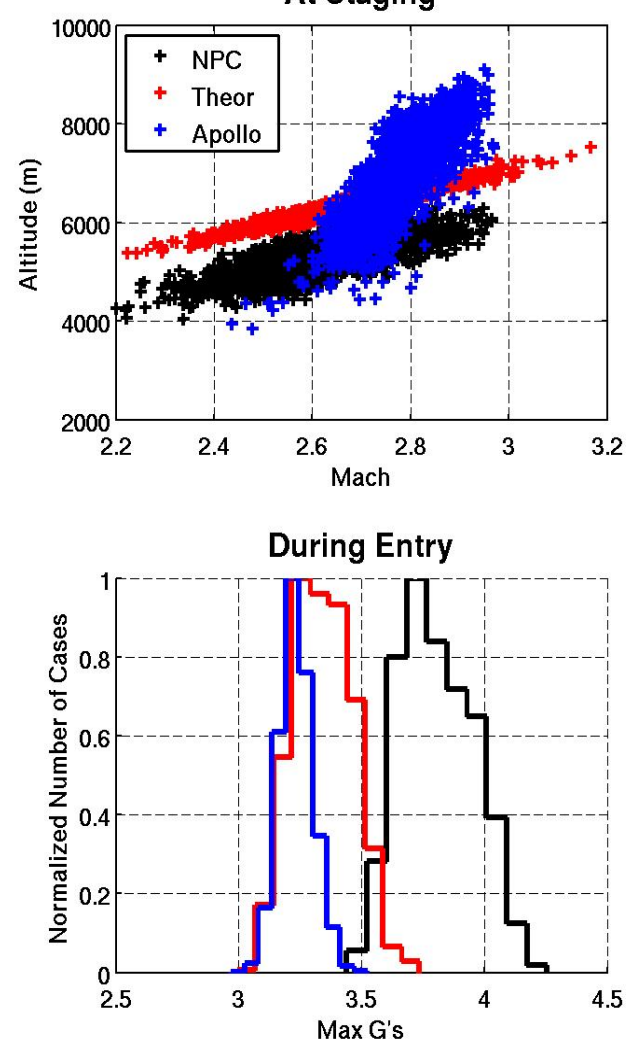

Touchdown

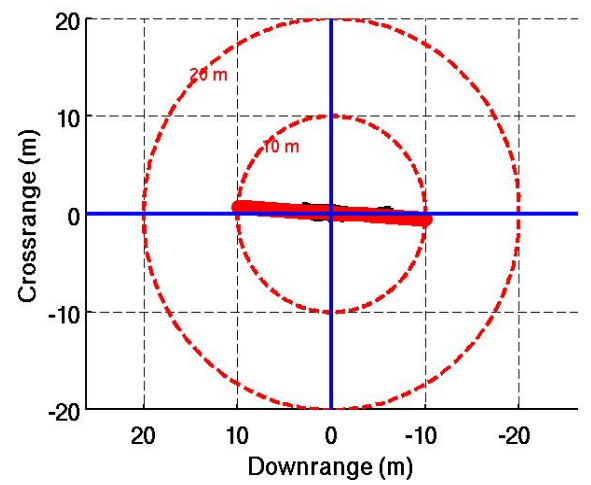

Touchdown

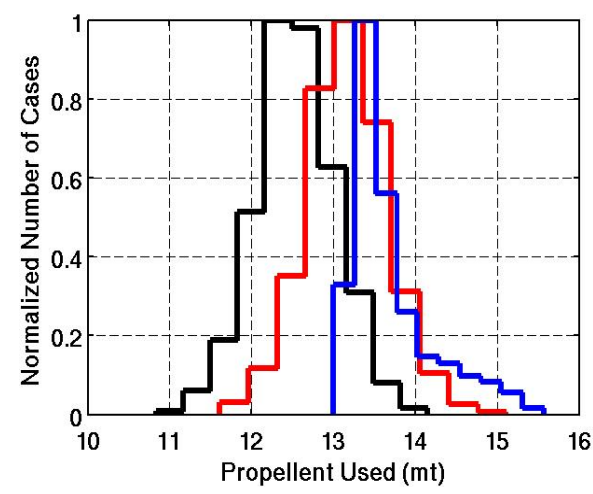

Figure 2. Monte Carlo results for mid-L/D aeroshell architecture.

architecture in that the same trends exist in the design parameter comparisons and the touchdown accuracy of $10 \mathrm{~m}$ are met for the three guidance algorithms. Also, the propellant usage variations at touchdown are similar between the NPC and APC guidance algorithms and the mean fuel usages compare to within $0.75 \mathrm{mT}$ or about $8 \%$ of the theoretical guidance. 

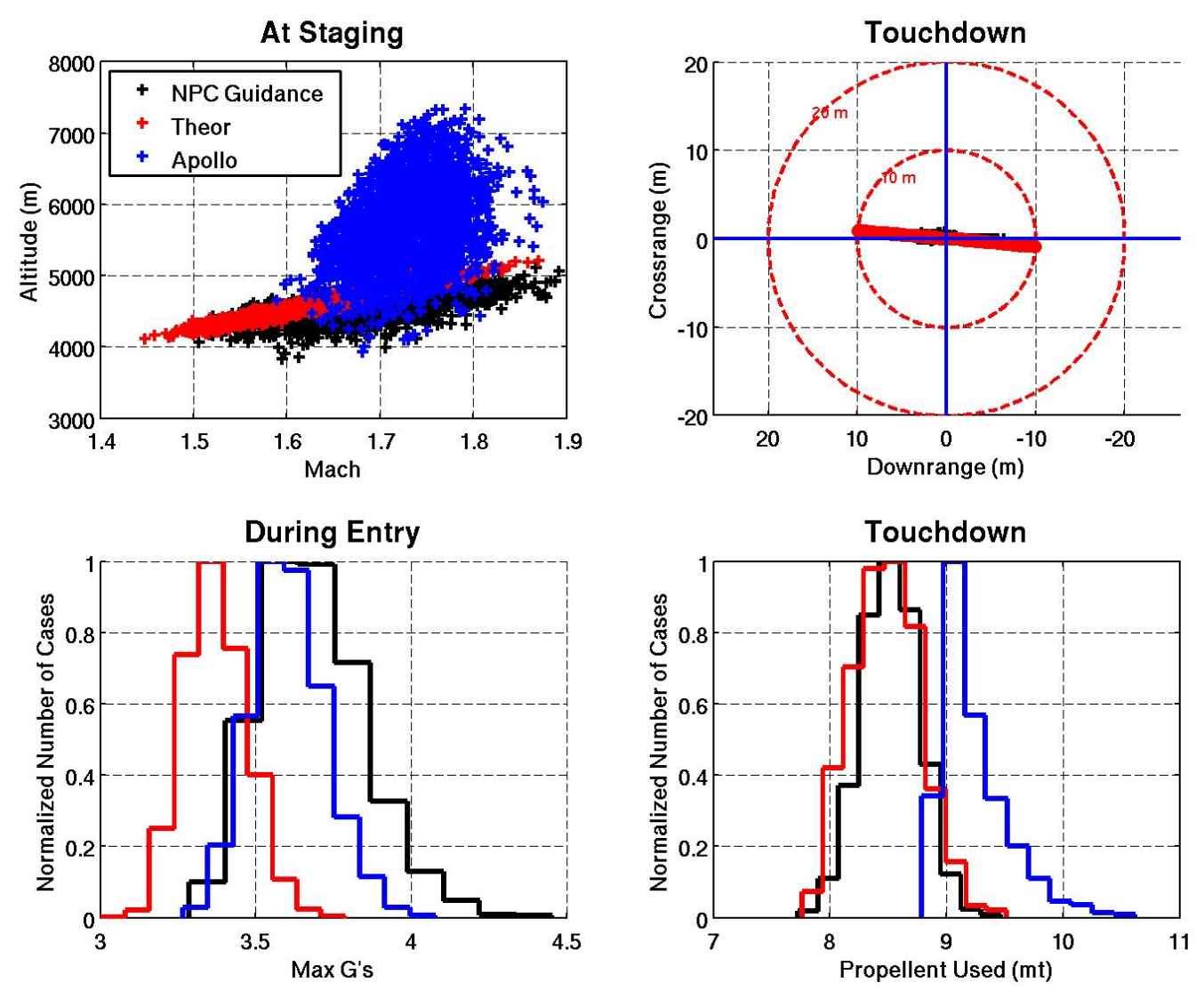

Figure 3. Monte Carlo results for HIAD architecture.

\section{B. Control Strategy Comparison}

Nominal trajectories were run using the EDL-SA POST2 EDL simulation to illustrate the differences in the two control strategies, bank angle and CG offset control, shown in Fig. 4 and 5. Figure 4 shows an example of the mid$\mathrm{L} / \mathrm{D}$ aeroshell architecture altitude-velocity profile for reference along with the heading error in degrees. This nominal trajectory was generated using the theoretical EDL guidance. The pullout phase contains the bank reversal strategy based on the allowable heading error (crossrange) as a function of velocity and when the error exceeds the allowable error, a bank reversal is commanded. The heading alignment phase beings at a specified velocity, about $2000 \mathrm{~m} / \mathrm{s}$ for this architecture, in which the bank angle is a function of the heading error and is driven to zero degrees at the start of terminal descent engine initiation. An example nominal trajectory was also run with the NPC guidance algorithm utilizing the CG offset control strategy for the HIAD architecture, shown in Fig. 5. Figure 5 illustrates the change in CG location, for both vertical $\left(\mathrm{z}_{\mathrm{CG}}\right)$ and lateral $\left(\mathrm{y}_{\mathrm{CG}}\right)$, throughout the trajectory. The $\mathrm{z}_{\mathrm{CG}}$ location provides the direct control of $\mathrm{L} / \mathrm{D}$ to meet range and flight path angle requirements, while the $\mathrm{y}_{\mathrm{CG}}$ location provides control of sideslip angle to minimize heading error directly. Improved tuning of the NPC guidance algorithm will reduce the non-linearity of the $\mathrm{z}_{\mathrm{CG}}$ movement in Fig. 5. Figures 6 and 7 show the initial Monte Carlo (2000-dispersed cases) results comparison of the bank angle and CG control strategies, in terms of conditions at staging or engine initiation and remaining propellant at touchdown. The powered descent delivery conditions in Fig. 6 show little variation (approximately $500 \mathrm{~m}$ difference in the mean) in altitude and relative velocity in both control strategies. The remaining propellant shown in Fig. 7 has a larger mean and slightly smaller variation for the bank angle control compared to the CG control, however the differences are small and the trends in the statistical data are similar, illustrating the feasibility for both methods. 


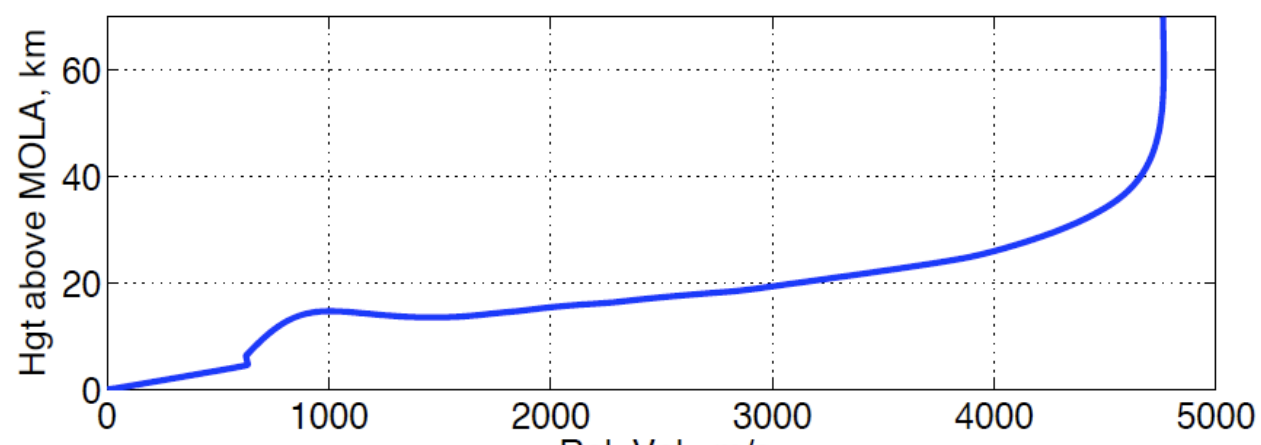

Rel. Vel., $\mathrm{m} / \mathrm{s}$

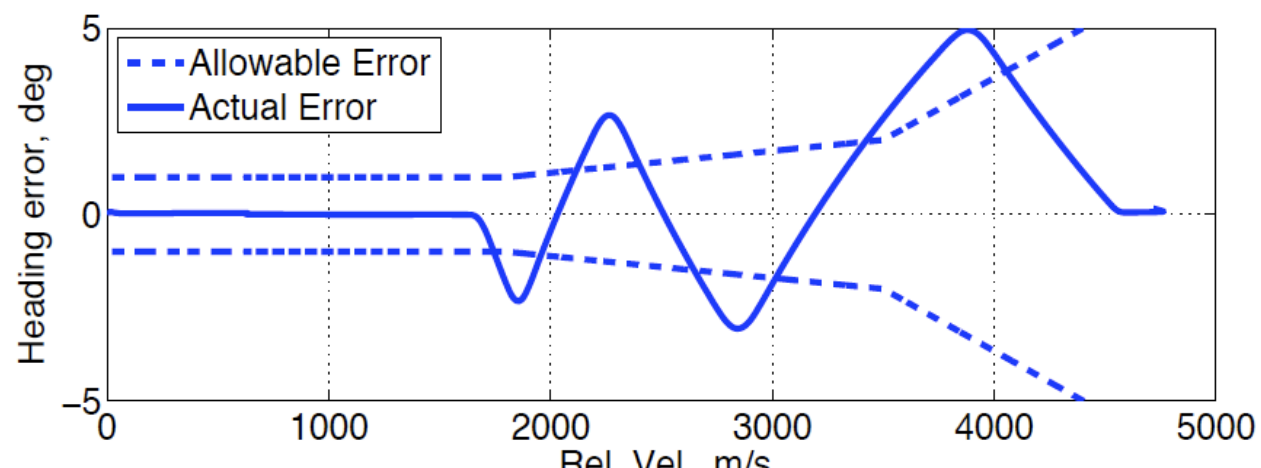

Rel. Vel., $\mathrm{m} / \mathrm{s}$

Figure 4. Nominal trajectory using bank angle control.

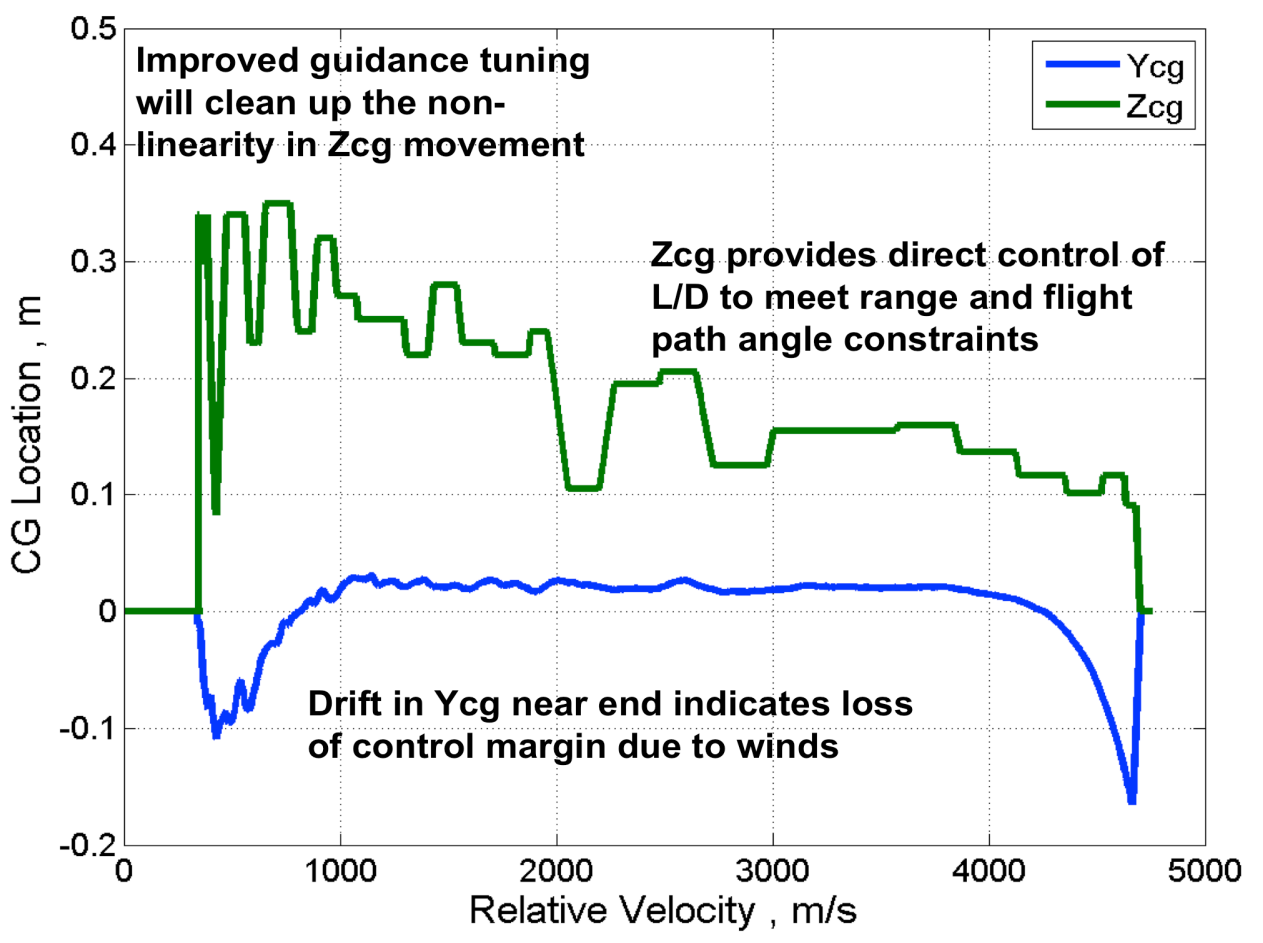

Figure 5. Nominal trajectory using CG offset control. 


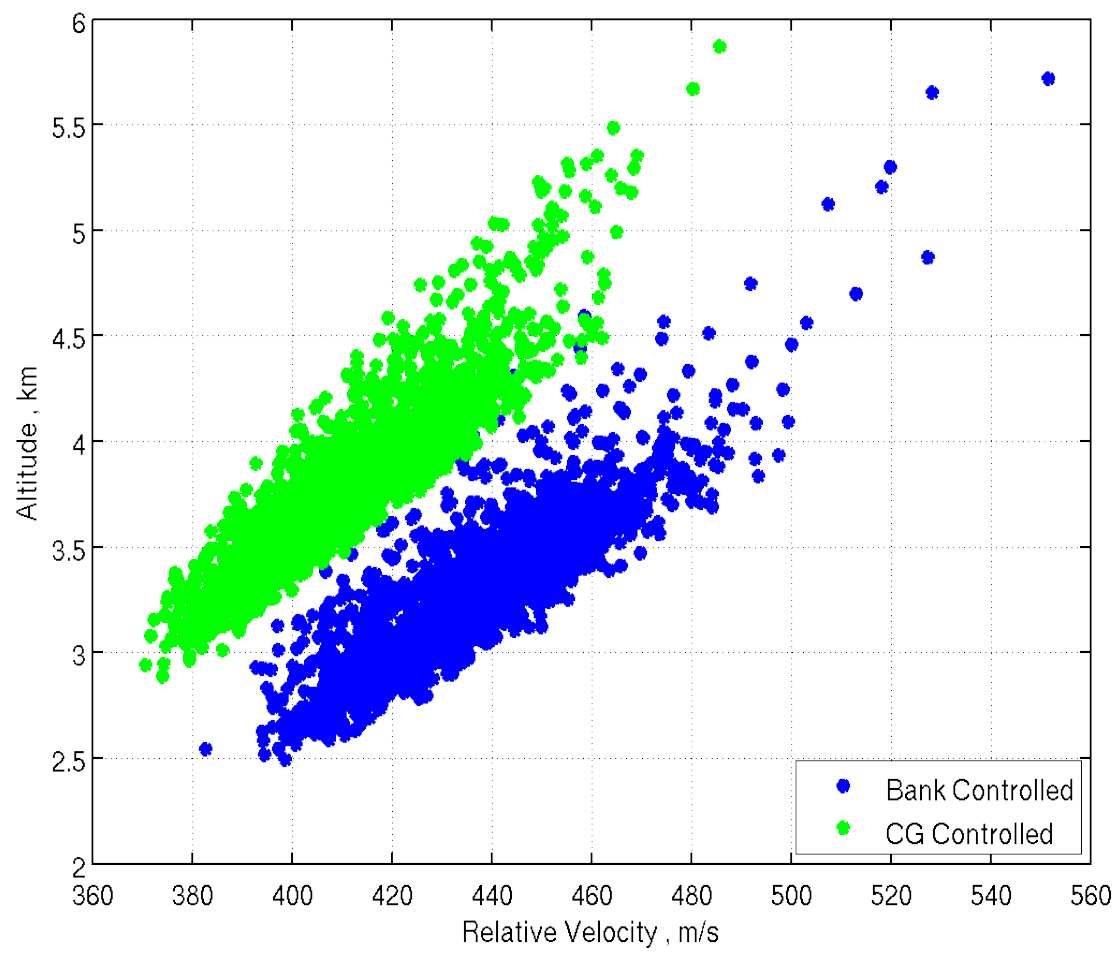

Figure 6. Monte Carlo CG vs. bank angle control at staging event.
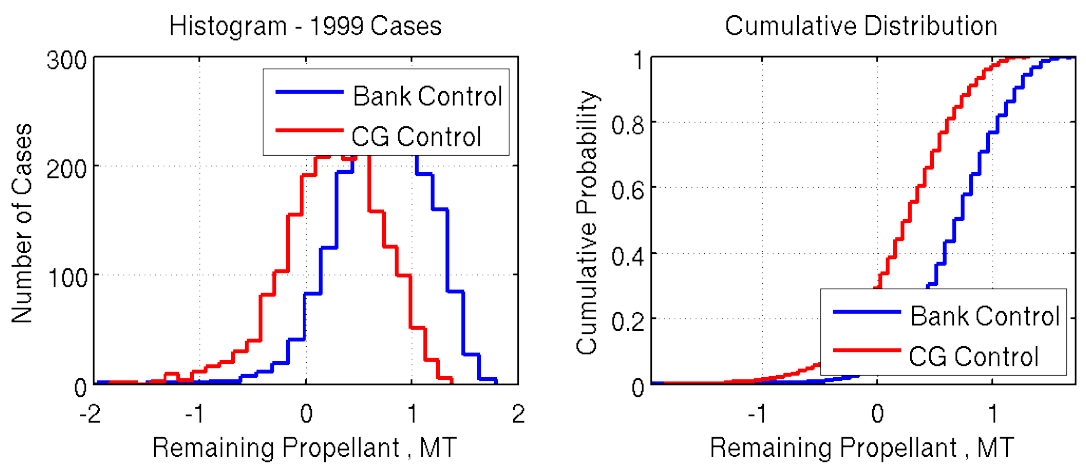

Statistics for

Remaining Propellant, MT

Bank Control:

Statistics for

Remaining Propellant, MT CG Control:

Mean $=0.71627$
1-Sigma $=0.42938$
3-Sigma $=1.2881$
Minimum $=-1.962$
$00.13 \%$-tile $=-1.4091$
$50.00 \%$-tile $=0.73566$
$99.87 \%$-tile $=1.6862$
Maximum $=1.7912$

Mean $=0.26122$
1-Sigma $=0.46198$
3-Sigma $=1.3859$
Minimum $=-1.8441$
$00.13 \%$-tile $=-1.3984$
$50.00 \%$-tile $=0.2877$
$99.87 \%$-tile $=1.2832$
Maximum $=1.3794$

Figure 7. Monte Carlo CG vs. bank angle control propellant.

8

American Institute of Aeronautics and Astronautics 092407 


\section{Conclusions}

Nominal trajectories and Monte Carlo results of the HIAD and mid-L/D aeroshell candidate technologies, using the three different guidance algorithms (theoretical, NPC and APC) and two control strategies (CG offset and bank angle), were generated using the Mars EDL-SA POST2 EDL simulation. It is shown in the Monte Carlo results presented that both the non-theoretical guidance algorithms (APC and NPC) can provide the required targeting accuracy of $10 \mathrm{~m}$ error at touchdown and have minimal impact on the design parameters of interest (maximum gloading, maximum aerothermal heat rate and load, staging conditions, etc) independent of the candidate aeroshell technologies or architectures. It is also shown from the nominal trajectories and initial Monte Carlo results that, specifically for the HIAD, both forms of entry control (bank angle and CG offset) appear to be feasible based on terminal descent delivery conditions and fuel usage trends at touchdown. CG control can vary the acceleration in the vertical and lateral channels simultaneously, allowing greater flexibility in guidance formulation. Bank control's constant lift acceleration is limiting in the guidance design, which can address cross track error or total range error separately, but not simultaneously. Lastly, more development (progression to 6DOF simulation), investigation and testing are needed to quantify the performance of both forms of entry control as well as fine-tuning of the higher fidelity guidance algorithms.

\section{References}

${ }^{1}$ Powell, R. W., Striepe, S. A., Desai, P. N., Queen, E. M., Tartabini, P. V., Brauer, G.L., Cornick, D. E., Olson, D. W., Petersen, F. M., Stevenson, R., Engle, M. C., and Marsh, S. M., "Program to Optimize Simulated Trajectories (POST2), Vol. II Utilization Manual.” Version 1.1.1G, May 2000.

${ }^{2}$ C.G. Justus D. L. Johnson "Mars Global Reference Atmospheric Model 2001 Version (Mars-GRAM 2001): Users Guide" NASA TM 2001-210961 April 2001

${ }^{3}$ Cianciolo, A. D., Davis, J. L., Powell, R. W., and Shidner, J. D., "Entry, Descent and Landing Systems Analysis: Exploration Class Simulation Overview and Results," AIAA/AAS Astrodynamics Specialist Conference, Toronto, August 2010.

${ }^{4}$ Powell, Richard W., "Numerical Roll Reversal Predictor Corrector Aerocapture and Precision Landing Guidance Algorithms for the Mars Surveyor Program 2001 Missions," AIAA 1998.

${ }^{5}$ Moseley, P. E., "The Apollo Entry Guidance: A Review of the Mathematical Development and Its Operational Characteristics", TRW Note No. 69-FMT-791, TRW, December 1, 1969.

${ }^{6}$ Sostaric, R. R. and Rea, J. R., "Powered Descent Guidance Methods For The Moon and Mars", AIAA Guidance, Navigation, and Control Conference and Exhibit 15 - 18 August 2005, San Francisco, California, paper AIAA 2005-6287. 